\title{
ERRATA, VOLUME 78
}

William Parry and Peter Walters, Endomorphisms of a Lebesgue space, pp. 272-276.

In reference 9, after the title should appear II.

In the last institution it should read "Mathematics Institute" instead of "Department of Mathematics."

Bang-yen Chen and Gerald D. Ludden, Rigidity theorems for surfaces in euclidean space, pp. 72-73.

p. 73 , line 20 , " $K \geqq 0$ " should read " $K=$ constant $\geqq 0$,"

line 25 , " $E^{m}$ " should read " $E^{5}$,"

line 29, "space" should read "5-space." 